\title{
Dimerization of Polycyclic Aromatic Hydrocarbon Molecules and Radicals under Flame Conditions
}

\author{
Qian Mao, ${ }^{\dagger}$ Dingyu Hou, ${ }^{\dagger}$ Kai H. Luo, ${ }^{*},{ }^{\dagger} \ddagger \odot$ and Xiaoqing You ${ }^{\dagger}$
}

${ }^{\dagger}$ Center for Combustion Energy, Key Laboratory for Thermal Science and Power Engineering of Ministry of Education, Department of Energy and Power Engineering, Tsinghua University, Beijing 100084, China

${ }^{\ddagger}$ Department of Mechanical Engineering, University College London, Torrington Place, London WC1E 7JE, U.K.

Supporting Information

\begin{abstract}
This work presents a dynamic and kinetic study on the dimerization of polycyclic aromatic hydrocarbon (PAH) molecules and radicals under flame conditions using reactive force field (ReaxFF) molecular dynamics (MD) simulations. The accuracy of the ReaxFF force field is evaluated through comparing with quantum chemistry (QC) calculations of the barrier heights and species concentrations of PAHs reacting with $\mathrm{H}$ and $\mathrm{OH}$ radicals. A series of homobinary collisions between $\mathrm{PAH}$ molecules/radicals are performed to reveal the influence of temperature, molecular size, PAH composition, and the number of radical sites on the dynamics and kinetics of PAH dimerization. Instead of directly forming the strong covalent bonds, the majority of the binary collisions between $\mathrm{PAH}$ radicals are bound with weak intermolecular interactions. Effects of oxygen on PAH

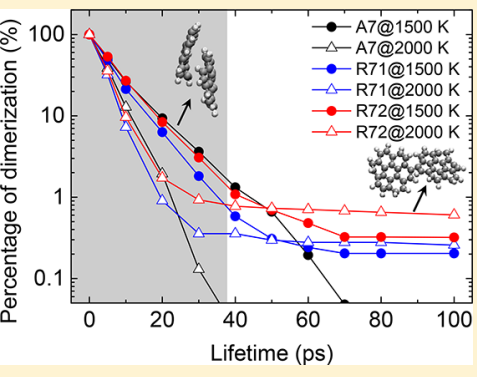
radical dimerization are also investigated, which indicates that the oxygenated $\mathrm{PAH}$ radicals are less likely to contribute to soot nucleation. In addition, the temperature, PAH characteristic, and radical site dependent collision efficiency for PAH radical-radical combinations is extracted from this study.
\end{abstract}

\section{INTRODUCTION}

Incomplete combustion of hydrocarbon fuels under fuel-rich conditions leads to the generation of the carbonaceous particulates known as soot. ${ }^{\text {In }}$ addition to its adverse effect on engine fuel efficiency, soot is regarded as one of the main environment contaminants, which poses a significant risk to human lives. ${ }^{2}$ Studies of soot formation have been performed for many practical and laboratory-scale combustion systems, and it is commonly accepted that soot formation experiences the following stages: aromatic formation, soot nucleation, coagulation, and surface growth. ${ }^{3}$ Among them, soot nucleation is a critical step yet still poorly understood. According to the previous experimental study, ${ }^{4}$ the soot nucleation pathway is through physical $\mathrm{PAH}$ dimerization to stacks. However, both theoretical ${ }^{5-7}$ and experimental ${ }^{8}$ studies report that the intermolecular interaction between $\mathrm{PAH}$ molecules is too weak to stabilize the PAH dimers at flame temperatures. Recent studies on soot nucleation have shifted from physical dimerization to chemical dimerization as PAHs are connected by covalent bonds. ${ }^{9,10}$ Elvati et al. ${ }^{11}$ proposed the role of radical-radical combinations in nascent soot formation and evaluated the sensitivity of soot nucleation to collision efficiency. And they ${ }^{11}$ also reported the oxygen chemistry has a significant impact on the chemical growth of soot. Moreover, Johansson et al. ${ }^{12}$ proposed the clustering of hydrocarbons by a radical-chain reaction (CHRCR) mechanism.

In the model of homodimerization of PAH molecules/ radicals, the reaction rates are estimated from the collision theory by taking into account the specific flame temperature and collision efficiency. ${ }^{10,13,14}$ The collision efficiency for PAH radical-radical combinations is a parameter introduced to account for the reversibility, which is usually given arbitrarily and independent of temperature and PAH characteristic. ${ }^{10,11}$ Therefore, an accurate collision efficiency for PAH molecules/ radicals is essential.

Quantum chemistry (QC) calculations of large $\mathrm{PAH}$ molecules/radicals are still challenging due to the prohibitively high computational cost despite impressive progress in computing hardware and software in recent decades. To simulate such large systems, some have tried to use a force field approach instead of the QC method. ${ }^{6,15,16}$ ReaxFF is a bond order based force field and parametrized against data from QC calculations, which is capable of describing chemically reacting systems without a priori knowledge of predefinition of reactive sites. ${ }^{17}$ ReaxFF-based reactive molecular dynamics (MD) has matured over the past decade and become a powerful yet affordable tool for the development of kinetic mechanisms for systems of large molecules and complex reactions. ${ }^{18-20}$

The objectives of this study are threefold: first, to assess the reliability of the ReaxFF MD method in describing reactive systems composed of $\mathrm{PAH}$ and $\mathrm{H}, \mathrm{OH}$ radicals by comparing against $\mathrm{QC}$ calculation based kinetic studies; second, to understand the dimerization mechanism of PAH molecules/ radicals and extract the temperature and $\mathrm{PAH}$ characteristic dependent collision efficiency for radical-radical combina-

Received: July 24, 2018

Revised: October 17, 2018

Published: October 18, 2018 
Table 1. Reaction Rate Parameters in the Chemical Kinetic Model ${ }^{a}$

\begin{tabular}{|c|c|c|c|c|c|}
\hline no. & reactions & $A$ & $n$ & $E(\mathrm{kcal} / \mathrm{mol})$ & re \\
\hline $\mathrm{r} 1$ & $\mathrm{~A} 7+\mathrm{H} \rightarrow \mathrm{R} 71+\mathrm{H}_{2}$ & $5.60 \times 10^{9}$ & 1.656 & 0.9986 & 24 \\
\hline$-\mathrm{r} 1$ & $\mathrm{R} 71+\mathrm{H}_{2} \rightarrow \mathrm{A} 7+\mathrm{H}$ & $1.0 \times 10^{12}$ & 0.545 & 0.8618 & 28 \\
\hline $\mathrm{r} 2$ & $\mathrm{R} 71+\mathrm{H} \rightarrow \mathrm{R} 72+\mathrm{H}_{2}$ & $5.14 \times 10^{9}$ & 1.656 & 0.9986 & 24 \\
\hline$-\mathrm{r} 2$ & $\mathrm{R} 72+\mathrm{H}_{2} \rightarrow \mathrm{R} 71+\mathrm{H}$ & $1.0 \times 10^{12}$ & 0.545 & 0.8618 & 28 \\
\hline r3 & $\mathrm{R} 72+\mathrm{H} \rightarrow \mathrm{R} 73+\mathrm{H}_{2}$ & $4.67 \times 10^{9}$ & 1.656 & 0.9986 & 24 \\
\hline$-r 3$ & $\mathrm{R} 73+\mathrm{H}_{2} \rightarrow \mathrm{R} 72+\mathrm{H}$ & $1.0 \times 10^{12}$ & 0.545 & 0.8618 & 28 \\
\hline r4 & $\mathrm{R} 73+\mathrm{H} \rightarrow \mathrm{R} 74+\mathrm{H}_{2}$ & $4.20 \times 10^{9}$ & 1.656 & 0.9986 & 24 \\
\hline$-\mathrm{r} 4$ & $\mathrm{R} 74+\mathrm{H}_{2} \rightarrow \mathrm{R} 73+\mathrm{H}$ & $1.0 \times 10^{12}$ & 0.545 & 0.8618 & 28 \\
\hline r5 & $\mathrm{A} 7+\mathrm{OH} \rightarrow \mathrm{R} 71+\mathrm{H}_{2} \mathrm{O}$ & $9.63 \times 10^{11}$ & 0.503 & 0.1043 & 29 \\
\hline$-\mathrm{r} 5$ & $\mathrm{R} 71+\mathrm{H}_{2} \mathrm{O} \rightarrow \mathrm{A} 7+\mathrm{H}_{2} \mathrm{O}$ & $1.0 \times 10^{9}$ & 0.647 & 0.3722 & \\
\hline r6 & $\mathrm{R} 71+\mathrm{OH} \rightarrow \mathrm{R} 72+\mathrm{H}_{2} \mathrm{O}$ & $8.83 \times 10^{11}$ & 0.503 & 0.1043 & 29 \\
\hline$-\mathrm{r} 6$ & $\mathrm{R} 72+\mathrm{H}_{2} \mathrm{O} \rightarrow \mathrm{R} 71+\mathrm{OH}$ & $1.0 \times 10^{9}$ & 0.647 & 0.3722 & p.w \\
\hline r7 & $\mathrm{R} 72+\mathrm{OH} \rightarrow \mathrm{R} 73+\mathrm{H}_{2} \mathrm{O}$ & $8.03 \times 10^{11}$ & 0.503 & 0.1043 & 29 \\
\hline$-\mathrm{r} 7$ & $\mathrm{R} 73+\mathrm{H}_{2} \mathrm{O} \rightarrow \mathrm{R} 72+\mathrm{OH}$ & $1.0 \times 10^{9}$ & 0.647 & 0.3722 & \\
\hline r8 & $\mathrm{R} 73+\mathrm{OH} \rightarrow \mathrm{R} 74+\mathrm{H}_{2} \mathrm{O}$ & $7.22 \times 10^{11}$ & 0.503 & 0.1043 & 29 \\
\hline$-r 8$ & $\mathrm{R} 74+\mathrm{H}_{2} \mathrm{O} \rightarrow \mathrm{R} 73+\mathrm{OH}$ & $1.0 \times 10^{9}$ & 0.647 & 0.3722 & \\
\hline r9 & $\mathrm{A} 7+\mathrm{O} \rightarrow \mathrm{R} 71+\mathrm{OH}$ & $8.0 \times 10^{13}$ & 0.0 & 0.8897 & 30 \\
\hline $\mathrm{r} 10$ & $\mathrm{R} 71+\mathrm{O} \rightarrow \mathrm{R} 72+\mathrm{OH}$ & $7.33 \times 10^{13}$ & 0.0 & 0.8897 & 30 \\
\hline r11 & $\mathrm{R} 72+\mathrm{O} \rightarrow \mathrm{R} 73+\mathrm{OH}$ & $6.67 \times 10^{13}$ & 0.0 & 0.8897 & 30 \\
\hline $\mathrm{r} 12$ & $\mathrm{R} 73+\mathrm{O} \rightarrow \mathrm{R} 74+\mathrm{OH}$ & $6.00 \times 10^{13}$ & 0.0 & 0.8897 & 30 \\
\hline r13 & $\mathrm{R} 71+\mathrm{R} 71 \rightarrow \mathrm{P} 11$ & $5.94 \times 10^{42}$ & -8.83 & 0.8375 & 31 \\
\hline r14 & $\mathrm{R} 71+\mathrm{R} 72 \rightarrow \mathrm{P} 12$ & $1.19 \times 10^{43}$ & -8.83 & 0.8375 & 31 \\
\hline $\mathrm{r} 15$ & $\mathrm{R} 71+\mathrm{R} 73 \rightarrow \mathrm{P} 13$ & $1.78 \times 10^{43}$ & -8.83 & 0.8375 & 31 \\
\hline r16 & $\mathrm{R} 71+\mathrm{R} 74 \rightarrow \mathrm{P} 14$ & $2.38 \times 10^{43}$ & -8.83 & 0.8375 & 31 \\
\hline r17 & $\mathrm{R} 72+\mathrm{R} 73 \rightarrow \mathrm{P} 23$ & $3.56 \times 10^{43}$ & -8.83 & 0.8375 & 31 \\
\hline $\mathrm{r} 18$ & $\mathrm{OH}+\mathrm{OH} \rightarrow \mathrm{H}_{2} \mathrm{O}+\mathrm{O}$ & $3.97 \times 10^{4}$ & 2.4 & -0.1277 & 30 \\
\hline r19 & $\mathrm{H}+\mathrm{H}+\mathrm{M} \rightarrow \mathrm{H}_{2}+\mathrm{M}$ & $1.78 \times 10^{18}$ & -1.0 & 0.0000 & 30 \\
\hline
\end{tabular}

${ }^{a}$ p.w. $=$ Rate constants assigned in the present model are based on the forward rate constants of $\mathrm{A} 7+\mathrm{OH} \rightarrow \mathrm{A} 7 \bullet+\mathrm{H}_{2} \mathrm{O}$ reported in ref 29 and the equilibrium constants calculated at the B3LYP/6-311G $(\mathrm{d}, \mathrm{p})$ level. A7 represents the coronene molecule, and R7X represents the coronene radical with $\mathrm{X}$ equal to the total number of radical sites.

tions; and, finally, to evaluate the importance of the radicalradical combination to $\mathrm{PAH}$ growth and soot nucleation.

\section{METHODOLOGY}

2.1. Quantum Chemistry Calculation. According to the transition state theory, the barrier height of a reaction is the key factor to determine the reaction rate constant. In this study, geometries of reactants and transition states of hydrogen abstraction from $\mathrm{PAH}$ and $\mathrm{PAH}$ radicals by $\mathrm{H}$ and $\mathrm{OH}$ radicals are optimized by the density functional B3LYP method with the basis set $6-311 G(d, p){ }^{21}$ which has been widely used in previous studies involving chemical reactions of PAHs. ${ }^{2-24}$ Transition states are confirmed to connect the reactants and the products by inspection of the normal modes of the corresponding imaginary frequencies. On the basis of the optimized geometries, single point energies are then calculated using the density functional theory (DFT) methods of M06$2 \mathrm{X}^{25}$ and B3LYP with the 6-311G(d,p) basis set. All of the QC calculations are carried out using the Gaussian 09 software package. $^{26}$

2.2. Chemical Kinetic Modeling. A chemical reaction mechanism for reactions between $\mathrm{PAHs}$ and $\mathrm{H}, \mathrm{OH}$ radicals is built including 19 species and 27 reactions in total. Details can be found in Table 1. Reaction rate parameters in the form of the Arrhenius equation are derived on the basis of QC calculations and the transition state theory. Coronene, one of the common PAHs, is selected for study. Specifically, R1 in Table 1 represents the reaction of an $\mathrm{H}$ atom on a coronene molecule (A7) abstracted by a $\mathrm{H}$ radical. $\mathrm{R} 2$ means the successive hydrogen abstraction takes place on R71 to form an A7 diradical named R72. Here, the different structures of R72 are not differentiated due to the fact that the per-site rate constants of $\mathrm{H}$ abstraction from $\mathrm{A} 7$ and $\mathrm{A} 7$ radicals are similar according to our recent study ${ }^{24}$ at the level of M06-2X/6$311 \mathrm{G}(\mathrm{d}, \mathrm{p})$. Moreover, simplified reacting systems of $\mathrm{PAH}$ and $\mathrm{H}$ and $\mathrm{PAH}$ and $\mathrm{OH}$ mixtures are then investigated in a $0-\mathrm{D}$ homogeneous reactor model at constant volume and temperature. Chemical kinetic modelings are performed using the Chemkin-Pro software ${ }^{27}$ to evaluate the kinetics of the formation of $\mathrm{PAH}$ radicals through hydrogen abstraction by $\mathrm{H}$ and $\mathrm{OH}$ radicals.

2.3. ReaxFF MD Simulations. The ReaxFF force field utilized in this study is the most up-to-date version involving interactions among $\mathrm{C}, \mathrm{H}$, and $\mathrm{O}$, which are derived through training against DFT calculations based on the B3LYP/6$311 G(d, p)$ method. $^{32-34}$ The van der Waals interaction for carbon is additionally described by the PBE exchangecorrelation functional with the DFT-D2 parameters. ${ }^{35,36}$ This force field has been successfully applied to investigate thermal fragmentation of fullerene, ${ }^{36}$ defect formation of graphene, ${ }^{37}$ PAH dimerization and nucleation, ${ }^{6,7}$ carbon nanotube nucleation from hydrocarbon precursors, ${ }^{38}$ etc. Moreover, prediction of the potential energy surface (PES) of PAH dimers by the current ReaxFF force field is only $2-3 \mathrm{kcal} / \mathrm{mol}$ lower than that by the DFT method of M06-2X, while the predictions of the classical force field in the form of Buckingham $^{1}$ and Lennard-Jones formulations ${ }^{39}$ are about 8 
$\mathrm{kcal} / \mathrm{mol}$ lower. Details of the parameters of the force field are given in the Supporting Information in Table S1.

Reaction barriers of $\mathrm{H}$ abstraction from $\mathrm{PAH}$ molecules/ radicals by $\mathrm{H}$ and $\mathrm{OH}$ radicals are calculated using the ReaxFF force field on the basis of the optimized geometries obtained in section 2.1. Afterward, the kinetics of the chemical reactions between $\mathrm{PAH}$ and $\mathrm{H} / \mathrm{OH}$ radicals are studied by ReaxFF MD simulations under the same initial conditions with the chemical kinetic modeling demonstrated in section 2.2.

Pyrene (A4) and coronene (A7) are simple but common precursors for soot inception. ${ }^{2}$ Thus, investigation of the PAH dimerization is performed using the above two PAH molecules and their radicals, namely, R41, R42, R71, and R72 shown in Figure 1. Besides, the R71_O radical is also considered in our

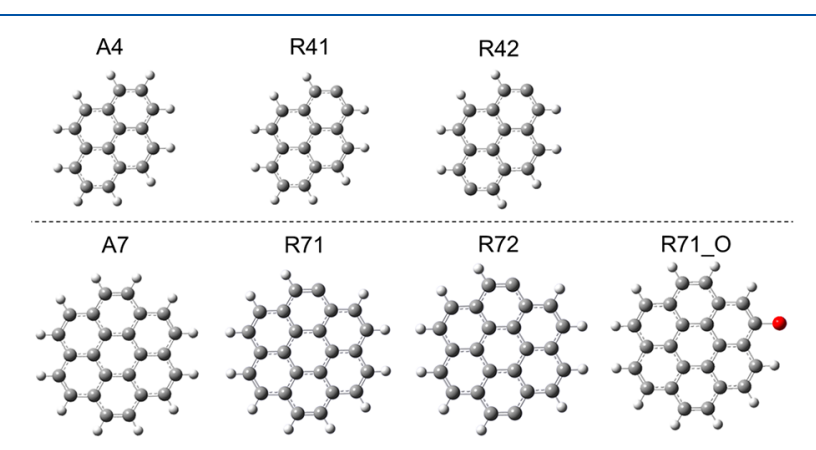

Figure 1. Representative PAH molecules/radicals utilized for studying the PAH dimerization.

study, as the oxygen has a significant impact in the chemical growth of soot. ${ }^{11}$ Before studying the binary collisions, structural equilibrium of $\mathrm{PAH}$ molecules and radicals is performed in the canonical ensemble (NVT) at temperatures of 1500,2000 , and $2500 \mathrm{~K}$, respectively. Constant temperature is maintained by the Nosé-Hoover thermostat with a damping constant of 100 fs. ${ }^{40}$ Afterward, binary collisions between PAH molecules/radicals are performed adiabatically in the microcanonical ensemble (NVE) with a time step of 0.1 fs to ensure the energy conservation during the collisions, which is similar to our previous studies. ${ }^{7,41}$ To achieve statistical significance, a total of 120,000 binary collisions are performed in the present study. Specifically, the initial relative mass center distance between the two colliding PAH molecules/radicals ranges from
50 to $80 \AA$, which is much larger than the effective intermolecular interaction distance between PAHs. ${ }^{7}$ The relative translational and rotational stochastic velocities are sampled from a Gaussian distribution. The impact parameter of the colliding molecules/radicals is sampled from 0 to $\sqrt{\varepsilon} d_{\mathrm{p}}$, where $\varepsilon$ is the enhancement factor for PAH dimerization ${ }^{7,42}$ and $d_{\mathrm{p}}$ is the collision diameter of a colliding molecule. ${ }^{13}$ The orientation of a molecule is rotated from 0 to $360^{\circ}$ in three axes. In this study, MD simulations are performed using the Large-scale Atomic/Molecular Massively Parallel Simulator (LAMMPS) package ${ }^{43}$ implemented with reax/c. Snapshots and movies in this study are prepared by Visual Molecular Dynamics (VMD). ${ }^{44}$

\section{RESULTS AND DISCUSSION}

On the basis of the above simulation methods, reliability of the ReaxFF MD method in describing reacting systems containing $\mathrm{PAH}$ molecules/radicals and $\mathrm{H}, \mathrm{OH}$ radicals is evaluated by QC calculation based kinetic studies in section 3.1. Section 3.2 discusses the dynamics of homodimerization of $\mathrm{PAH}$ molecules/radicals and extracts the collision efficiency for radical-radical combinations through forming covalent bonds. Finally, section 3.3 assesses the contribution of the radicalradical combination to the PAH growth and soot nucleation.

3.1. Chemical Kinetics. Table 2 illustrates the structure of the A7 molecule and the barrier heights of hydrogen abstraction from the A7 molecule and A7 radicals by $\mathrm{H}$ and $\mathrm{OH}$ radicals. The barrier heights at different $\mathrm{C}-\mathrm{H}$ sites are calculated by two DFT methods, namely, B3LYP/6-311G(d,p) and M06-2X/6-311G(d,p), and the ReaxFF force field. It is found that the barrier heights predicted by the ReaxFF force field situate between the results from B3LYP/6-311G(d,p) and M06-2X/6-311G(d,p), with deviations around 1 and $-4.5 \mathrm{kcal}$ $\mathrm{mol}^{-1}$, respectively. Our previous study indicates that the M06$2 \mathrm{X} / 6-311 \mathrm{G}(\mathrm{d}, \mathrm{p})$ is found to obtain reaction barriers for $\mathrm{H}$ abstraction from PAHs closest to the high-level $a b$ initial method CCSD(T)/CBS among 11 tested DFT methods. ${ }^{24}$ For hydrogen abstraction from coronene molecule by $\mathrm{OH}$ radicals, the van der Waals complex $(\mathrm{A} 7 \cdots \mathrm{OH})$ is typically formed first as discussed extensively by Shiroudi and Deleuze. ${ }^{45}$ For simplicity, we assume the barrier height for hydrogen abstraction from $\mathrm{A} 7$ by $\mathrm{OH}$ radical is equal to the energy difference between its complex and the sum of A7 and

Table 2. Barrier Heights of Hydrogen Abstraction from Coronene (A7) by $\mathbf{H}$ and $\mathrm{OH}$ Radicals Calculated Using DFT Methods (B3LYP/6-311G(d,p) and M06-2X/6-311G(d,p)) and the ReaxFF Force Field (Units: kcal mol $\left.{ }^{-1}\right)^{a}$

\begin{tabular}{|c|c|c|c|c|c|c|c|}
\hline 3, & \multirow{2}{*}{$\begin{array}{l}\text { B3LYP/6- } \\
311 \mathrm{G}(\mathrm{d}, \mathrm{p})\end{array}$} & \multirow{2}{*}{$\begin{array}{l}\text { M06-2X/6- } \\
311 \mathrm{G}(\mathrm{d}, \mathrm{p})\end{array}$} & \multirow{2}{*}{ ReaxFF } & \multicolumn{2}{|c|}{$\begin{array}{c}\text { B3LYP deviation from } \\
\text { M06-2X }\end{array}$} & \multicolumn{2}{|c|}{$\begin{array}{c}\text { ReaxFF deviation from } \\
\text { M06-2X }\end{array}$} \\
\hline & & & & $\begin{array}{c}\text { absolute } \\
\text { differences }\end{array}$ & $\begin{array}{c}\text { relative } \\
\text { percentage }\end{array}$ & $\begin{array}{c}\text { absolute } \\
\text { differences }\end{array}$ & $\begin{array}{c}\text { relative } \\
\text { percentage }\end{array}$ \\
\hline $\mathrm{A} 7+\mathrm{H} \rightarrow \mathrm{R} 71+\mathrm{H}_{2}$ & 11.2 & 16.8 & 12.5 & -5.6 & $-33.3 \%$ & -4.3 & $-25.6 \%$ \\
\hline $\mathrm{R} 71+\mathrm{H} \rightarrow \mathrm{R} 72(12)+\mathrm{H}_{2}$ & 12.1 & 17.6 & 13.2 & -5.5 & $-31.3 \%$ & -4.4 & $-25.0 \%$ \\
\hline $\mathrm{R} 71+\mathrm{H} \rightarrow \mathrm{R} 72(14)+\mathrm{H}_{2}$ & 11.4 & 17.0 & 12.5 & -5.6 & $-32.9 \%$ & -4.5 & $-26.5 \%$ \\
\hline $\mathrm{R} 71+\mathrm{H} \rightarrow \mathrm{R} 72(15)+\mathrm{H}_{2}$ & 11.3 & 17.0 & 12.5 & -5.7 & $-33.5 \%$ & -4.5 & $-26.5 \%$ \\
\hline $\mathrm{R} 71+\mathrm{H} \rightarrow \mathrm{R} 72(16)+\mathrm{H}_{2}$ & 11.4 & 17.3 & 12.6 & -5.9 & $-34.1 \%$ & -4.7 & $-27.2 \%$ \\
\hline $\mathrm{R} 71+\mathrm{H} \rightarrow \mathrm{R} 72(17)+\mathrm{H}_{2}$ & 11.4 & 17.1 & 12.6 & -5.7 & $-33.3 \%$ & -4.0 & $-23.4 \%$ \\
\hline $\mathrm{A} 7+\mathrm{OH} \rightarrow \mathrm{R} 71+\mathrm{H}_{2} \mathrm{O}$ & -0.9 & 2.5 & -1.7 & -3.4 & - & -4.2 & - \\
\hline
\end{tabular}

${ }^{a}$ Radicals with one abstracted site and two abstracted sites are named as $\mathrm{R} 71$ and $\mathrm{R} 72$ (xy), respectively [R72(xy) represents a coronene radical of two radical sites with a sequence number of $x$ and $y$, as shown in the inserted snapshot]. 
$\mathrm{OH}$ radical, which is negative, as listed in Table 2. Generally, the barrier heights of $\mathrm{H}$ abstraction reactions by $\mathrm{H}$ and $\mathrm{OH}$ radicals at different sites predicted by the ReaxFF force field have only slight deviation from DFT calculations, which indicates the reliability of the ReaxFF force field to describe a system with PAHs and radicals. Furthermore, it is noteworthy that the computational cost for a single point energy by the ReaxFF force field is at least 100,000 times faster than the DFT calculations, which enables some complex systems to be studied.

Afterward, we compare the kinetics of reactions between $\mathrm{PAH}$ molecules and $\mathrm{H} / \mathrm{OH}$ radicals. Figure 2 shows the mole

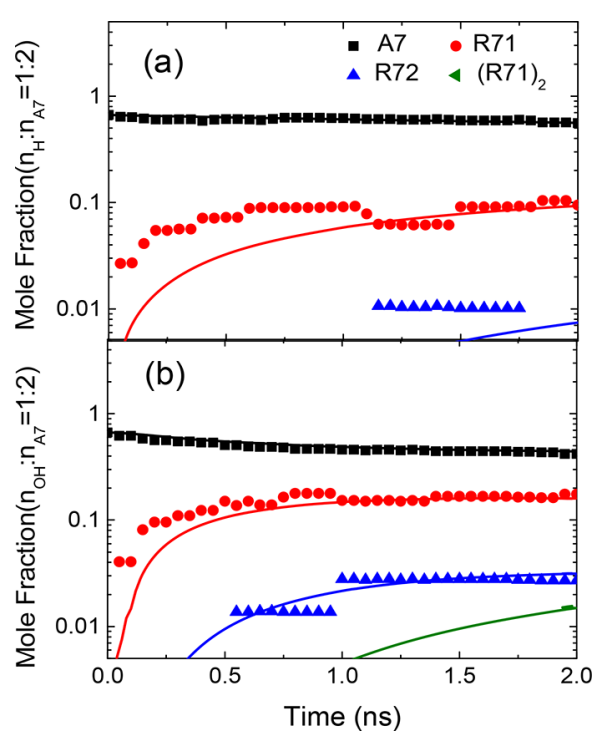

Figure 2. Mole fractions of species versus time in a 0-D homogeneous reactor at constant volume $\left(100 \times 100 \times 100 \AA^{3}\right)$ and temperature $(1500 \mathrm{~K})$ : (a) A7 and $\mathrm{H}$ radical system; (b) A7 and $\mathrm{OH}$ radical system. The dots are results from ReaxFF MD, and the solid lines are from chemical kinetic modeling.

fractions of species in a 0 -D homogeneous reactor at the constant volume and temperature with initial molecule fractions of $x_{\mathrm{H}(\mathrm{OH})}=0.33$ and $x_{\mathrm{A} 7}=0.66$, respectively. The mole fractions of reactants and key products from the ReaxFF $\mathrm{MD}$ simulation are shown in dots, and those from kinetic modeling are shown in solid lines. Though there is some deviation especially at the beginning due to the discrete nature of MD simulations, results from MD simulations globally agree well with those from the chemical kinetic modeling. According to Figure 2, it is found that hydrogen abstraction from PAHs by $\mathrm{OH}$ radicals is faster than that by $\mathrm{H}$ radicals. Formation of species R71 is the most predominant, and then, species R72 follows with two $\mathrm{H}$ abstractions. It is noted that the mole fraction of (R71) 2 (R71 dimer connected by covalent bonds) is not negligible in the system composed of PAH and $\mathrm{OH}$ radicals according to the chemical kinetic modeling, which differs greatly from the ReaxFF MD simulation. Similar results happen for systems with initial molecule concentrations ranging from 0.125 to $0.913 \mathrm{~mol} / \mathrm{cm}^{3}$. In fact, the collision efficiency of $\mathrm{PAH}$ radical-radical combinations is given arbitrarily in the range $0.1-0.85$ in the chemical kinetic models, ${ }^{10,11,31}$ and it is desirable to determine a more precise value for radical-radical combinations.

3.2. Dimerization of PAHs. To obtain the collision efficiency for $\mathrm{PAH}$ radical-radical combinations through forming covalent bonds, a series of binary collisions need to be studied. Initially, binary collisions between homo PAH molecules/radicals are studied at temperatures ranging from 1500 to $2500 \mathrm{~K}$. In this study, the products from both the molecule-molecule and radical-radical dimerization are uniformly named as dimers. Firstly, representative dynamics including collision, dimerization, combination, and decomposition of PAH molecules, radicals at $2000 \mathrm{~K}$ are displayed in Figure 3. For the A7 molecule, the formed dimer is quite unstable, as it falls apart into molecules with a lifetime of about 10 ps according to Figure 3a. For PAH radicals, such as R71 radicals, they travel and rotate around each other after collision, as seen in Figure 3b. Subsequently, a covalent bond is formed as the two radical sites come closer, which represents the combination of the PAH radicals. However, the majority of the dimers composed of R71 radicals cannot take combinations through forming covalent bonds before the dimer falls apart, as illustrated in Figure 3c. Furthermore, as demonstrated in Figure 3d, the edge-to-edge collision is more conducive to producing a dimer connected by the covalent bond.

Through examining the behaviors of PAH molecules and $\mathrm{PAH}$ radicals after binary collisions, quantitative studies, such as the lifetime distribution of PAH dimer and the collision efficiency of the PAH radical combination, are scrutinized. The lifetime of a dimer is evaluated from the moment of dimer formation to that of the dimer decomposition. For PAH dimers formed through intermolecular interactions, the interlayer displacement ranges from 3.5 to $3.9 \AA \AA^{46}$ and our previous study indicated that the criterion of $4 \AA$ is acceptable to distinguish PAH dimers from molecules, ${ }^{6}$ which is also used in this study. Parts a-c of Figure 4 summarize the percentage of dimerization from identical PAH molecules/radicals versus the lifetime. First, behaviors of dimerization of A4, A7, and R71_O are quite similar, as the percentage of dimerization has an exponential correlation with the corresponding dimer lifetime shown in Figure 4a. Globally, the lifetime of PAH dimer decreases with the increasing temperature, and is sensitive to both the molecular size and composition. Because of the stronger intermolecular interactions between A7 molecules than that of the A4 molecules, ${ }^{2,47}$ the A7 dimer has a longer lifetime than the A4 dimer at the same temperature. Though R71 O and A7 have a high similarity in size, the lifetime of the R71_O dimer is slightly shorter than the A7 dimer. This observation agrees with the result from Violi and co-workers, ${ }^{11}$ as the oxygenated PAH prohibits the dimerization to some extent. According to Figure $4 \mathrm{~b}$ and $\mathrm{c}$, the relationship between the percentage of $\mathrm{PAH}$ radical dimerization and the lifetime of $\mathrm{PAH}$ dimer is markedly different from that of the PAH molecules, such as A4 and A7 in Figure 4a. That is, two remarkably distinctive relations are observed. Initially, the lifetime of $\mathrm{PAH}$ radical dimer has a negative correlation with temperature in the first $20-30$ ps shown in Figure $4 \mathrm{~b}$ and $\mathrm{c}$, which is similar to the behaviors of A4, A7, and R71_O in Figure 4a. This indicates that those PAH dimers are bound with intermolecular interactions. Afterward, the percentage of $\mathrm{PAH}$ radical dimerization remains mostly constant versus dimer lifetime at 1500 and $2000 \mathrm{~K}$ due to the formation of covalent bonds through radical-radical combinations. Contrary to the negative temperature dependent relation for the physical dimerization, the percentage of $\mathrm{PAH}$ dimerization through forming covalent bonds at $2000 \mathrm{~K}$ exceeds that at $1500 \mathrm{~K}$. Even though the R71 radicals have a higher possibility of forming a covalent bond than R41 radicals, 


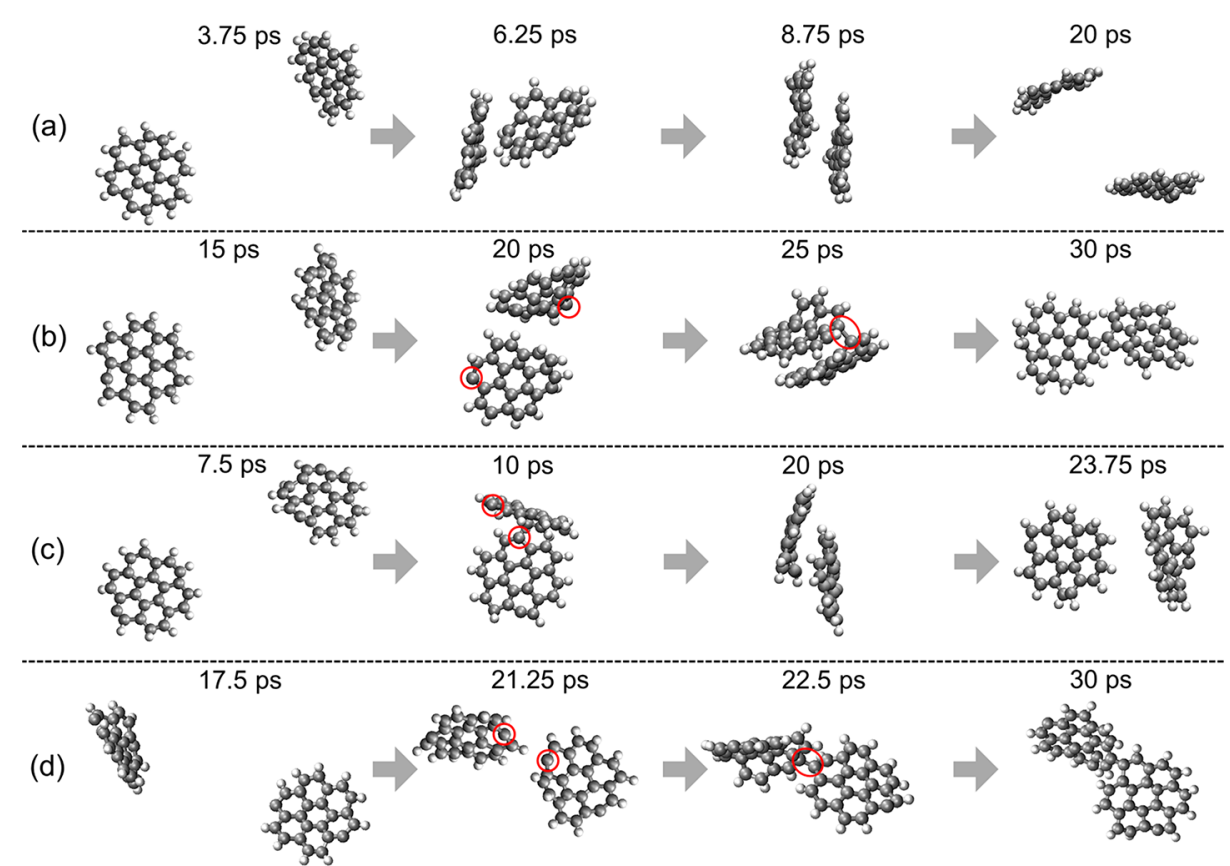

Figure 3. Dynamics of homomolecular binary collisions involving (a) A7 molecules, (b) R71 radicals, (c) R71 radicals, and (d) R72 radicals in different collision orientations and velocities. All of the molecules and radicals in the above four configurations are equilibrated at $2000 \mathrm{~K}$. The radical sites and the formed covalent bonds are highlighted in red circles ( $\mathrm{C}$ atom, gray; $\mathrm{H}$ atom, white).
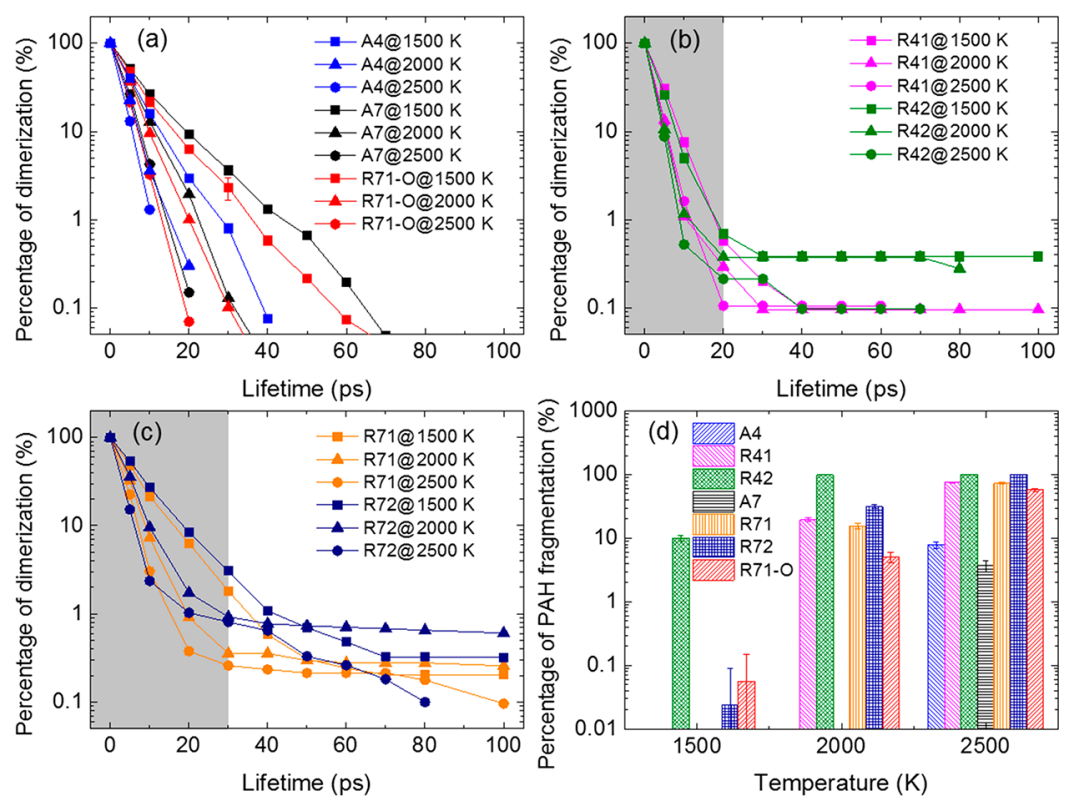

Figure 4. Changes in the percentage of homodimerization of PAH molecules/radicals versus dimer lifetime at different temperatures: (a) A4, A7, and R71_O; (b) R41 and R42; (c) R71 and R72; (d) percentages of the PAH molecule/radical fragmentation after binary collisions.

it is still much lower compared to the suggested collision efficiency in previous studies. ${ }^{10,11}$ Figure $4 \mathrm{~d}$ illustrates the percentages of fragmentation of PAHs after binary collisions at different temperatures. It is found that, when the temperature rises to $2500 \mathrm{~K}$, nearly all of the $\mathrm{R} 72$ and R42 radicals fragment, which leads to the percentage of dimerization decreasing instead of remaining constant, as shown in Figure $4 \mathrm{~b}$ and $\mathrm{c}$ at $2500 \mathrm{~K}$. This agrees with the previous experimental and theoretical studies of thermal fragmentation of PAHs at about $2200 \mathrm{~K}^{6,48}$ It is noteworthy that the thermal stability decreases with the increasing radical site and decreasing $\mathrm{PAH}$ radical size. That is, the percentage of the decomposition of
R42 is higher than that of R41 and R72 at the same temperature.

Additionally, heterodimerization between PAH radicals is investigated. Percentages of the dimerization versus lifetime of $\mathrm{PAH}$ dimer from binary collision between an R71_O radical and a R71 radical at temperatures of 1500, 2000, and $2500 \mathrm{~K}$ are presented in Figure 5. It is found that the relation between the dimerization percentage and temperature is similar to that of the homodimerization of R71_O radicals, as illustrated in Figure 4a. Instead of forming covalence bonds, the dimers formed by the above two radicals are associated with intermolecular interactions. This once more confirms the 


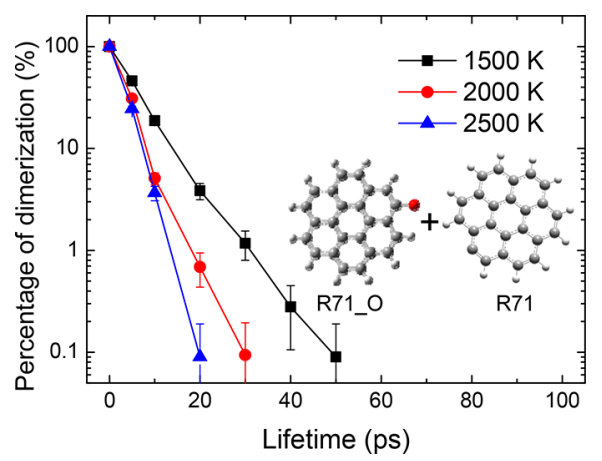

Figure 5. Percentage of the dimerization from heteromolecular binary collisions between a R71 radical and a R71_O radical with respect to dimer lifetime.

difficulty of radical-radical combinations, as it is limited to certain types of PAHs. ${ }^{11}$

3.3. Radical-Radical Combination Model. On the basis of the $\mathrm{PAH}$ dimerization and the $\mathrm{PAH}$ radical combination discussed in section 3.2, it is found that only the PAH dimers formed from the $\mathrm{PAH}$ radicals through the radical-radical combination possess a long lifetime for later PAH growth and soot nucleation. Thus, a simple kinetic model is established to evaluate the contribution from $\mathrm{PAH}$ radical combinations to soot nucleation based on the collision theory ${ }^{2,10,11,13}$

$$
\begin{aligned}
& \left\{\begin{array}{l}
\frac{\mathrm{d}\left[n_{\text {radical }}\right]}{\mathrm{d} t}=-2 \cdot k\left[n_{\text {radical }}\right]^{2} \\
\frac{\mathrm{d}\left[n_{\text {dimer }}\right]}{\mathrm{d} t}=k\left[n_{\text {radical }}\right]^{2}
\end{array}\right. \\
& {\left[n_{\text {radical }}\right]_{t=0}=n_{0}, \quad\left[n_{\text {dimer }}\right]_{t=0}=0} \\
& k=\beta \varepsilon \sqrt{\frac{4 \pi k_{\mathrm{B}} T}{m_{\mathrm{p}}}} d_{\mathrm{p}}^{2}\left[n_{\text {radical }}\right]^{2}
\end{aligned}
$$

where $\beta$ is the collision efficiency extracted from radicalradical combinations from Figure 4 . For example, $\beta$ for R41 is roughly equal to 0.001 and $\mathrm{R} 71$ is 0.003 at $2000 \mathrm{~K}$. The collision efficiency $(\beta=0.1)$ for PAH radical combinations in the study of Violi and co-workers ${ }^{11}$ is selected and adopted for comparison. The typical concentration of A4 is about $10^{13}$ $10^{14} \mathrm{~cm}^{-3}$, and that for A7 is $10^{11}-10^{12} \mathrm{~cm}^{-3}$ as detected in flames, ${ }^{2}$ while the concentration of the PAH radicals is $1-2$ orders of magnitude smaller than their PAH molecules. ${ }^{10} \mathrm{On}$ the basis of the relation between the PAH radical and dimer expressed above, the normalized concentrations $\left(n / n_{0}\right)$ of the dimer from radical-radical combinations at varied initial concentrations are deduced and plotted as a function of time in Figure 6. Through applying the collision efficiency of $0.1,{ }^{11}$ the concentration of (R41) $)_{2}$ to R41 equals about 0.3 at an initial $\mathrm{R} 41$ concentration of $6.5 \times 10^{12} \mathrm{~cm}^{-3}$. However, it is about $1-$ 2 orders of magnitude smaller using the collision efficiency extracted from the current MD simulations under the same initial condition. The $(\mathrm{R} 41)_{2}$ to $\mathrm{R} 41$ is about 0.1 when the initial R41 concentration increases to $6.5 \times 10^{13} \mathrm{~cm}^{-3}$, which is acceptable to explain the soot nucleus concentration detected in experiments. ${ }^{10}$ The concentrations of $(\mathrm{R} 71)_{2}$ from R71 radicals at an initial number concentration of $6.5 \times 10^{10}$ and $6.5 \times 10^{11} \mathrm{~cm}^{-3}$ roughly equal $10^{7}$ and $10^{9} \mathrm{~cm}^{-3}$. This is too small to account for the observed soot nuclei at the

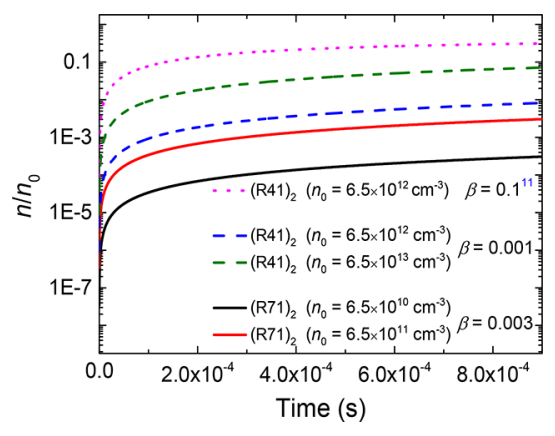

Figure 6. Normalized concentrations ( $n$ is the concentration of PAH dimer, and $n_{0}$ is the initial concentration of PAH radical) of PAH dimer formed from R41 and R71 at different initial concentrations and collision efficiencies (from our current study and Violi and coworkers ${ }^{11}$ ) as a function of time at $2000 \mathrm{~K}$.

concentration of $10^{11}-10^{13} \mathrm{~cm}^{-3} \cdot{ }^{22}$ Therefore, soot nucleation from radical-radical combinations is accessible to the situation where the concentration of PAH radical is high.

\section{CONCLUSIONS}

This study utilizes ReaxFF MD simulations to investigate the PAH molecule-molecule dimerization and the PAH radicalradical combination. Prediction of the energy barriers and species concentrations of PAHs reacting with $\mathrm{H}$ and $\mathrm{OH}$ radicals by the ReaxFF MD simulations is quantitatively consistent with those by quantum chemistry calculations based kinetic studies. To reveal the underlying relation among the lifetime of the $\mathrm{PAH}$ dimer, temperature, and $\mathrm{PAH}$ characteristic, a total of 120,000 binary collisions between two identical/ different PAH molecules/radicals are performed. Behaviors of homodimerization of PAH molecules, such as A4 and A7, and oxygenated $\mathrm{PAH}$ radicals, such as $\mathrm{R} 71 \_\mathrm{O}$, are similar, as the formed dimers are attracted by intermolecular interactions. The lifetime of these PAH dimers is found to decrease with increasing temperature and decreasing $\mathrm{PAH}$ size. Moreover, the molecular composition affects the lifetime of the PAH dimer as the existence of oxygen decreases its lifetime. For $\mathrm{PAH}$ radicals, such as R41 and R71, the majority of the binary collisions lead to the formation of $\mathrm{PAH}$ dimers bound with intermolecular interactions, which is similar to the dimers formed from PAH molecules. And few of them are connected through covalent bonds from radical-radical combinations after collision. Below the thermal fragmentation temperature thresholds of the PAH radicals, collision efficiencies of radicalradical combinations of pyrene and coronene are extracted from ReaxFF MD simulations. The collision efficiency becomes larger with increasing $\mathrm{PAH}$ size, radical site, and temperature. This study indicates that collision efficiency prescribed in previous studies was generally overestimated and the contribution from $\mathrm{PAH}$ radical combinations to $\mathrm{PAH}$ growth and soot nucleation is highly dependent on the initial radical concentration.

\section{ASSOCIATED CONTENT}

\section{S Supporting Information}

The Supporting Information is available free of charge on the ACS Publications website at DOI: 10.1021/acs.jpca.8b07102.

ReaxFF force field parameters for the $\mathrm{C} / \mathrm{H} / \mathrm{O}$ system (PDF) 


\section{AUTHOR INFORMATION}

\section{Corresponding Author}

*E-mail: K.Luo@ucl.ac.uk.

\section{ORCID $\odot$}

Kai H. Luo: 0000-0003-4023-7259

\section{Notes}

The authors declare no competing financial interest.

\section{ACKNOWLEDGMENTS}

Support from the National Natural Science Foundation of China (Grant Nos. 91441120, 51390493, and 91541122) is gratefully acknowledged. The simulations were partly performed on the Tsinghua High-Performance Parallel Computer supported by the Tsinghua National Laboratory for Information Science and Technology. Support from the UK EPSRC under the project "UK Consortium on Mesoscale Engineering Sciences (UKCOMES)" (Grant Nos. EP/ L00030X/1 and EP/R029598/1) is also acknowledged.

\section{REFERENCES}

(1) Miller, J. H.; Mallard, W. G.; Smyth, K. C. Intermolecular Potential Calculations for Polycyclic Aromatic Hydrocarbons. J. Phys. Chem. 1984, 88 (21), 4963-4970.

(2) Wang, H. Formation of Nascent Soot and Other CondensedPhase Materials in Flames. Proc. Combust. Inst. 2011, 33 (1), 41-67.

(3) Frenklach, M. Reaction Mechanism of Soot Formation in Flames. Phys. Chem. Chem. Phys. 2002, 4 (11), 2028-2037.

(4) Schuetz, C. A.; Frenklach, M. Nucleation of Soot: Molecular Dynamics Simulations of Pyrene Dimerization. Proc. Combust. Inst. 2002, 29 (2), 2307-2313.

(5) Wang, Q.-D.; Wang, J.-B.; Li, J.-Q.; Tan, N.-X.; Li, X.-Y. Reactive Molecular Dynamics Simulation and Chemical Kinetic Modeling of Pyrolysis and Combustion of N-Dodecane. Combust. Flame 2011, 158 (2), 217-226.

(6) Mao, Q.; van Duin, A. C. T.; Luo, K. H. Formation of Incipient Soot Particles from Polycyclic Aromatic Hydrocarbons: A ReaxFF Molecular Dynamics Study. Carbon 2017, 121, 380-388.

(7) Mao, Q.; Ren, Y.; Luo, K. H.; van Duin, A. C. T. Dynamics and Kinetics of Reversible Homo-Molecular Dimerization of Polycyclic Aromatic Hydrocarbons. J. Chem. Phys. 2017, 147 (23), 244305.

(8) Sabbah, H.; Biennier, L.; Klippenstein, S. J.; Sims, I. R.; Rowe, B. R. Exploring the Role of PAHs in the Formation of Soot: Pyrene Dimerization. J. Phys. Chem. Lett. 2010, 1 (19), 2962-2967.

(9) Violi, A.; Sarofim, A. F.; Truong, T. N. Mechanistic Pathways to Explain H/C Ratio of Soot Precursors. Combust. Sci. Technol. 2002, 174 (11-12), 205-222.

(10) Johansson, K. O.; Dillstrom, T.; Elvati, P.; Campbell, M. F.; Schrader, P. E.; Popolan-vaida, D. M.; Richards-Henderson, N.; Wilson, K.; Violi, A.; Michelsen, H. Radical-Radical Reactions, Pyrene Nucleation, and Incipient Soot Formation in Combustion. Proc. Combust. Inst. 2017, 36 (1), 799-806.

(11) Elvati, P.; Dillstrom, V. T.; Violi, A. Oxygen Driven Soot Formation. Proc. Combust. Inst. 2017, 36 (1), 825-832.

(12) Johansson, K. O.; Head-Gordon, M. P.; Schrader, P. E.; Wilson, K. R.; Michelsen, H. A. Resonance-Stabilized Hydrocarbon-Radical Chain Reactions May Explain Soot Inception and Growth. Science 2018, 361, 997-1000.

(13) Raj, A.; Sander, M.; Janardhanan, V.; Kraft, M. A Study on the Coagulation of Polycyclic Aromatic Hydrocarbon Clusters to Determine Their Collision Efficiency. Combust. Flame 2010, 157 (3), 523-534.

(14) Blanquart, G.; Pitsch, H. A Joint Vol--Surface-Hydrogen MultiVariate Model for Soot Formation. Combustion Generated Fine Carbonaceous Particles; Karlsruhe University Press: Villa Orlandi, Anacapri, Italy, 2007; pp 437-463.
(15) Chung, S. H.; Violi, A. Peri-Condensed Aromatics with Aliphatic Chains as Key Intermediates for the Nucleation of Aromatic Hydrocarbons. Proc. Combust. Inst. 2011, 33 (1), 693-700.

(16) Totton, T. S.; Misquitta, A. J.; Kraft, M. A Quantitative Study of the Clustering of Polycyclic Aromatic Hydrocarbons at High Temperatures. Phys. Chem. Chem. Phys. 2012, 14 (12), 4081-4094.

(17) Senftle, T.; Hong, S.; Islam, M. M.; Kylasa, S. B.; Zheng, Y.; Shin, Y. K.; Junkermeier, C.; Engel-Herbert, R.; Janik, M. J.; Aktulga, H. M.; et al. The ReaxFF Reactive Force-Field: Development, Applications, and Future Directions. npj Comput. Mater. 2016, 2, 15011.

(18) Chenoweth, K.; Van Duin, A. C. T.; Dasgupta, S.; Goddard, W. A. Initiation Mechanisms and Kinetics of Pyrolysis and Combustion of JP-10 Hydrocarbon Jet Fuel. J. Phys. Chem. A 2009, 113 (9), $1740-1746$.

(19) Weismiller, M. R.; Russo, M. F.; Van Duin, A. C. T.; Yetter, R. A. Using Molecular Dynamics Simulations with a ReaxFF Reactive Force Field to Develop a Kinetic Mechanism for Ammonia Borane Oxidation. Proc. Combust. Inst. 2013, 34 (2), 3489-3497.

(20) Mao, Q.; van Duin, A. C. T.; Luo, K. H. Investigation of Methane Oxidation by Palladium-Based Catalyst via ReaxFF Molecular Dynamics Simulation. Proc. Combust. Inst. 2017, 36 (3), 4339-4346.

(21) Stephens, P. J.; Devlin, F. J.; Chabalowski, C. F.; Frisch, M. J. $\mathrm{Ab}$ Initio Calculation of Vibrational Absorption and Circular Dichroism Spectra Using Density Functional Force Fields. J. Phys. Chem. 1994, 98 (45), 11623-11627.

(22) You, X.; Whitesides, R.; Zubarev, D.; Lester, W. A.; Frenklach, M. Bay-Capping Reactions: Kinetics and Influence on Graphene-Edge Growth. Proc. Combust. Inst. 2011, 33 (1), 685-692.

(23) Zhang, H. B.; You, X.; Wang, H.; Law, C. K. Dimerization of Polycyclic Aromatic Hydrocarbons in Soot Nucleation. J. Phys. Chem. A 2014, 118, 1287-1292.

(24) Hou, D.; You, X. Reaction Kinetics of Hydrogen Abstraction from Polycyclic Aromatic Hydrocarbons by H Atoms. Phys. Chem. Chem. Phys. 2017, 19 (45), 30772-30780.

(25) Zhao, Y.; Truhlar, D. G. The M06 Suite of Density Functionals for Main Group Thermochemistry, Thermochemical Kinetics, Noncovalent Interactions, Excited States, and Transition Elements: Two New Functionals and Systematic Testing of Four M06-Class Functionals and 12 Other Function. Theor. Chem. Acc. 2008, 120 (13), 215-241.

(26) Frisch, M.; Trucks, G. W.; Schlegel, H. B.; Scuseria, G. E.; Robb, M. A.; Cheeseman, J. R.; Scalmani, G.; Barone, V.; Mennucci, B.; Petersson, G. A.; et al. Gaussian 09, revision D.01; Gaussian, Inc.: Wallingford, CT, 2009.

(27) CHEMKIN-Pro, A.17.2; ANSYS React. Des.: San Diego, CA, 2016.

(28) Mebel, A. M.; Lin, M. C.; Yu, T.; Morokuma, K. Theoretical Study of Potential Energy Surface and Thermal Rate Constants for the C6H5+H-2 and C6H6+H Reactions. J. Phys. Chem. A 1997, 101 (17), 3189-3196.

(29) Shiroudi, A.; Deleuze, M. S.; Canneaux, S. Theoretical Study of the Oxidation Mechanisms of Naphthalene Initiated by Hydroxyl Radicals: The $\mathrm{O}_{2}$ Addition Reaction Pathways. Phys. Chem. Chem. Phys. 2015, 17 (20), 13719-13732.

(30) Wang, Y.; Raj, A.; Chung, S. H. A PAH Growth Mechanism and Synergistic Effect on PAH Formation in Counterflow Diffusion Flames. Combust. Flame 2013, 160 (9), 1667-1676.

(31) Richter, H.; Granata, S.; Green, W. H.; Howard, J. B. Detailed Modeling of PAH and Soot Formation in a Laminar Premixed Benzene/Oxygen/Argon Low-Pressure Flame. Proc. Combust. Inst. 2005, 30 (1), 1397-1404.

(32) Becke, A. D. Density-Functional Thermochemistry. III. The Role of Exact Exchange. J. Chem. Phys. 1993, 98 (7), 5648-5652.

(33) Lee, C.; Yang, W.; Parr, R. G. Development of the ColleSalvetti Correlation-Energy Formula into a Functional of the Electron Density. Phys. Rev. B: Condens. Matter Mater. Phys. 1988, 37 (2), 785-789. 
(34) Chenoweth, K.; van Duin, A. C. T.; Goddard, W. A. ReaxFF Reactive Force Field for Molecular Dynamics Simulations of Hydrocarbon Oxidation. J. Phys. Chem. A 2008, 112 (5), 1040-1053.

(35) Grimme, S. Semiempirical GGA-Type Density Functional Constructed with a Long-Range Dispersion Correction. J. Comput. Chem. 2006, 27 (15), 1787-1799.

(36) Srinivasan, S. G.; van Duin, A. C. T.; Ganesh, P. Development of a ReaxFF Potential for Carbon Condensed Phases and Its Application to the Thermal Fragmentation of a Large Fullerene. J. Phys. Chem. A 2015, 119 (4), 571-580.

(37) Yoon, K.; Rahnamoun, A.; Swett, J. L.; Iberi, V.; Cullen, D. A.; Vlassiouk, I. V.; Belianinov, A.; Jesse, S.; Sang, X.; Ovchinnikova, O. S.; et al. Atomistic-Scale Simulations of Defect Formation in Graphene under Noble Gas Ion Irradiation. ACS Nano 2016, 10 (9), 8376-8384.

(38) Khalilov, U.; Bogaerts, A.; Neyts, E. C. Atomic Scale Simulation of Carbon Nanotube Nucleation from Hydrocarbon Precursors. Nat. Commun. 2015, 6, 10306.

(39) van de Waal, B. W. Calculated Ground-State Structures of 13Molecule Clusters of Carbon Dioxide, Methane, Benzene, Cyclohexane, and Naphthalene. J. Chem. Phys. 1983, 79 (8), 3948-3961.

(40) Evans, D. J.; Holian, B. L. The Nose-Hoover Thermostat. J. Chem. Phys. 1985, 83 (8), 4069-4074.

(41) Mao, Q.; Luo, K. H. Trace Metal Assisted Polycyclic Aromatic Hydrocarbons Fragmentation, Growth and Soot Nucleation. Proc. Combust. Inst. 2018, 000, 1-8.

(42) Harris, S. J.; Kennedy, I. M. The Coagulation of Soot Particles with van Der Waals Forces. Combust. Sci. Technol. 1988, 59 (4-6), 443-454.

(43) Plimpton, S. Fast Parallel Algorithms for Short-Range Molecular Dynamics. J. Comput. Phys. 1995, 117 (1), 1-19.

(44) Humphrey, W.; Dalke, A.; Schulten, K. VMD: Visual Molecular Dynamics. J. Mol. Graphics 1996, 14 (1), 33-38.

(45) Shiroudi, A.; Deleuze, M. S. Theoretical Study of the Oxidation Mechanisms of Naphthalene Initiated by Hydroxyl Radicals: The $\mathrm{H}$ Abstraction Pathway. J. Phys. Chem. A 2014, 118 (20), 3625-3636.

(46) Kinney, C. R.; Nunn, R. C.; Walker, P. L. Carbonization of Anthracene and Graphitization of Anthracene Carbons. Ind. Eng. Chem. 1957, 49 (5), 880-884.

(47) Herdman, J. D.; Miller, J. H. Intermolecular Potential Calculations for Polynuclear Aromatic Hydrocarbon Clusters. J. Phys. Chem. A 2008, 112 (28), 6249-6256.

(48) Chen, T.; Gatchell, M.; Stockett, M. H.; Delaunay, R.; Domaracka, A.; Micelotta, E. R.; Tielens, A. G. G. M.; Rousseau, P.; Adoui, L.; Huber, B. A.; et al. Formation of $\mathrm{H} 2$ from Internally Heated Polycyclic Aromatic Hydrocarbons: Excitation Energy Dependence. J. Chem. Phys. 2015, 142 (14), 144305. 